\title{
The reward of great collaborations
}

\author{
Fiona Watt runs the Centre for Stem Cells and Regenerative Medicine at King's College London and is an outspoken \\ advocate for women scientists. Since April 2018, she has been on secondment as Executive Chair of the Medical \\ Research Council, one of the major funders of biomedical research in the UK.
}

\section{Fiona M. Watt}

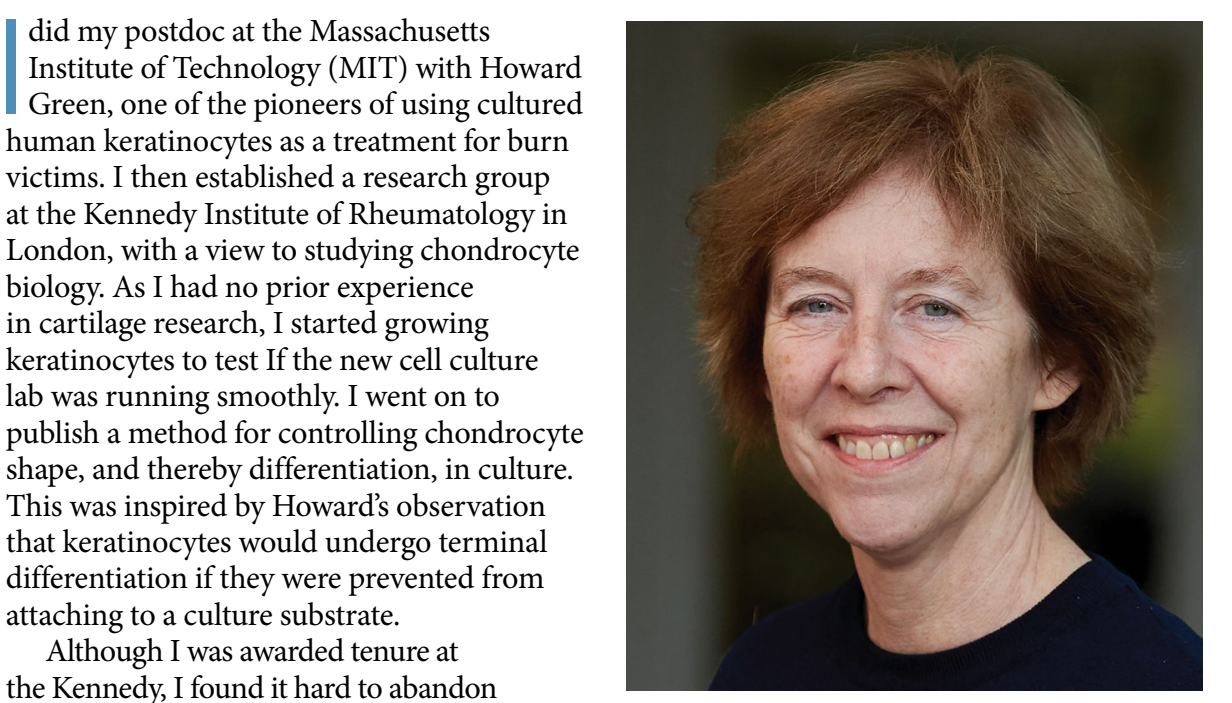

keratinocyte research and trying to stay abreast of two different fields was making me unhappy. Looking for a change I was invited to apply at the Imperial Cancer Research Fund (ICRF), a forerunner of the Francis Crick Institute. While interviewing there I met a fellow cell biologist, Charles O'Neill, who was also interested in cell shape. He described making petri dishes with patterns of circular palladium islands, each accommodating a single cell, and using them to examine the link between adhesion and anchorage-dependent growth in fibroblasts. We struck up a collaboration, and completed most of our experiments before I joined the ICRF. We mainly communicated by letter - I still have one in which Charles summarised our findings of the previous month and ended "Let's do another keratinocyte experiment with different times. Shall we see how we are situated in ten days ( $28^{\text {th }}$ April)?”

We submitted our paper to a highly respected journal but it was rejected on the grounds of lack of novelty. The decision letter was admirably straightforward, "There is ... a two to one vote against publication... and my policy... is to accept the majority verdict". The work was later published in the Proceedings of the National Academy of Sciences of the USA (Proc. Natl Acad. Sci. USA 85, 5576-5580; 1988), but I was deeply disappointed by that early rejection - 'anguished' best describes how I felt. Over the years I have received many rejection letters from different journals and I have come to realise that it is often our most innovative work that is rejected as 'lacking novelty'. Our 1988 paper showed that you could study stem-cell-niche interactions at single cell resolution in a quantitative way by varying one environmental signal at a time, but it was another ten years before the use of micro-patterned islands to explore cell fate decisions began to take off (Science 276, 1425-1428; 1997).

Although my early work fostered a careerlong interest in the signals that control the switch from epidermal stem cells to differentiated cells, it was not until 2007 that I returned to micro-patterning. At the time I was working in Cambridge, where I was introduced to Wilhelm Huck (now at the Radboud University in Nijmegen, the Netherlands), a polymer chemist by training. We hit it off immediately, Wilhelm establishing the basis of our collaboration as "if you don't mess with the chemistry, I won't mess with the biology". When we repeated the 1988 experiment, the micropatterning process was much simpler and we could quantitate differentiation much less subjectively than before. It was probably because of these advances that my postdoc, John Connelly (now a group leader at Queen Mary University of London), was genuinely surprised at how well the original experiment could be reproduced. Our interest reignited, we used micro-patterned substrates to dissect how the extrinsic stimulus of restricted cell spreading was transduced into transcriptional responses to determine epidermal stem cell fate (Nat. Cell Biol. 12, 711-718; 2010).

And what about 2018? We are still using micro-patterned substrates - and, yes, the original experiment is still reproducible - but now we study the kinetics of differentiation in individual cells using live cell reporters. Our current collaborators, Ignacio Bordeu and Gunnar Pruessner from Imperial College London, are mathematicians, who we met by chance through their work with a neighbouring lab. Their computer simulations are helping us to understand the experimental data but also to generate new, testable hypotheses. We also have the opportunity, as partners in the Human Cell Atlas initiative (www.humancellatlas.org), to apply thirty years of in vitro observations to elucidating cell state transitions in human skin. Now, our collaborators are the entire community of skin researchers worldwide.

So what are the lessons I have learnt in the past 30 years? First of all, patience; sometimes you have to put aside a problem until the technology catches up sufficiently to enable you to find a solution. Second, the power of inter-disciplinary collaboration; my best collaborations started through serendipity and flourished through common goals, mutual trust and affection. Third, persistence; my message to scientists who are suffering the anguish of a rejected paper is that you can always publish important work that others will find, read and cite regardless of the journal. That 1988 paper has enriched my career, and I sometimes wonder how different my research path might have been if I had not met Charles all those years ago.

Fiona M. Watt

King's College London Centre for Stem Cells and Regenerative Medicine, London, UK.

e-mail:Fiona.watt@kcl.ac.uk

Published online: 28 August 2018 https://doi.org/10.1038/s41556-018-0157-5

Competing interests

The author declares no competing interest. 